\title{
A Review on Video Compression and Embedding Techniques
}

\author{
K. Rajalakshmi \\ Ph.D. Research scholar \\ Dept. of Computer science and Engineering \\ Alagappa University, Karaikudi
}

\author{
K. Mahesh, PhD \\ Professor \\ Dept. of Computer science and Engineering \\ Alagappa University, Karaikudi
}

\begin{abstract}
Data embedding techniques embed the secret image into another image for increasing the privacy. The data embedding techniques can also be substituted to the videos so that the confidentiality of the image, video, and the embedded data can be maintained. In this paper, multiple compression techniques such as Principle Component Analysis (PCA) based method, Set Partitioning in Hierarchical Trees (SPIHT) algorithm and fuzzy concepts are analyzed. The embedding techniques are classified into two types such as digital image watermarking and data hiding algorithms. The digital watermarking techniques like Discrete Cosine Transform (DCT), Discrete Wavelet Transform (DWT) and Least Significant Bit (LSB) are surveyed. Further, the data hiding techniques such as H.264/AVC video stream and MPEG videos are analyzed. In the survey results, it is clear that the existing techniques do not efficiently restore the compressed image, the pixel information is lost during the transformations. Further, the existing techniques have increased time complexity and computational complexity.
\end{abstract}

\section{Keywords}

Principal Component Analysis (PCA), Set Partitioning in Hierarchical Trees (SPIHT) algorithm, Least Significant Bit (LSB), Discrete Wavelet Transform (DWT), Discrete Cosine Transform (DCT), Fuzzy.

\section{INTRODUCTION}

Digital video represents the visual images moving in the form of digital data. Whereas, the analog video represents the moving images in analog video format. Video compression is a technology used for transforming the video signals with the maintenance of the original quality under various situations such as storage constraint, time delay constraint and power constraint (Suganya.G 2014). By exploiting the data redundancy between consecutive frames and computational resources, the storage requirement is reduced. The existing techniques exploit the effective video compression techniques for reducing the file size with minimal impact on the visual quality. Multiple video codec standards and algorithms are used for transmitting the video in digital form. The compression techniques are classified into two broad categories such as,

\section{- Lossy compression}

- Lossless compression

The exploitation of lossy and lossless compression techniques for the video compression provides an optimal compression ratio than the traditional data compression standards. In lossy compression, the size of the video is greatly decreased. The lossy compression compresses the actual data from the video frame and replaces them with an approximation. Whereas, in the lossless compression, the actual quality of the video frames are maintained even after the compression process. The increased usage of digital content introduces multiple security issues like online data vulnerability and copyrights violation (Khan et al. 2014). Thus, to secure the video frames from possible security threats, the data hiding, and digital watermarking techniques are used. Data hiding is defined as the process of embedding the information inside another data source without altering the perceptual quality. It is a specialized way of hiding the messages because even the sender and the receiver don't realize the hidden messages. The data hiding technique converts the actual information in the original data into an alternative equivalent multimedia file such as audio, video or image. The alternative multimedia is in turn hidden within another object. The hidden message is then sent through the network to the receiver. On receiving the message, the receiver separates the actual message from the hidden message.

The digital watermarking technique is defined as a process of hiding the messages. The digital watermarking technique embeds the secret information and the extra information within the cover image. The embedded images are then extracted and used for various applications such as content protection, owner detection, authentication and copyright protection. In certain cases, the scaling factor is used for embedding the watermark in the cover image. By exploiting the digital watermarking techniques, the digital video can be protected from the various illegal users and the ownership rights can be given to the digital content. The key advantages of the digital watermarking techniques are depicted below,

- Robust

- Prevention against various attacks

- Ability to perform filtering, scaling, compression and cropping

There exist various digital watermarking techniques. Based on the robustness of the embedded watermark, the efficiency of the digital watermarking algorithms can be enhanced. The digital watermarking techniques contain two main components such as embedding algorithm and a detection algorithm. In the embedding algorithm, the cover image and the watermark image are embedded using a secret key.

Whereas, in the case of the detection process, the watermarked data is detected using a secret key. In this paper, the various compression techniques such as fuzzy concept, SPIHT algorithm, and PCA based method are analyzed. Further, the embedding techniques such as digital watermarking and data hiding algorithms are surveyed. From the survey results, it is clear that the existing compression techniques have increased time complexity, computational complexity, increased memory consumption. Further, the existing methods do not efficiently restore the compressed images and the pixel information is lost during the 
transformations. Hence, to address the issues of the existing image compression and embedding techniques a block code formation framework is proposed for an efficient video embedding.

The organization of the paper is as follows. Section II illustrates the compression techniques used for videos. Section III elaborates the various embedding techniques. Section IV describes the steps involved in the proposed technique and Section V concludes the paper.

\section{COMPRESSION TECHNIQUES}

Video Compression is the process of reducing the number of pixels in each frame by preventing the redundant information. The goal of the compression techniques is as follows,

- Reduce the color resolution and color nuances in the image

- Prevent the invisible portions of the image

- Compare the adjacent frames and remove the unchanged details between two images.

The algorithms and techniques used for the video compression are depicted in Fig.1.

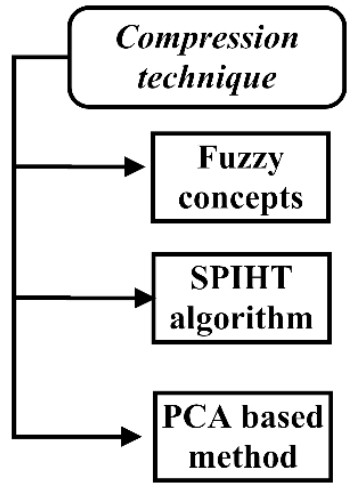

Fig 1: Categories of compression techniques

\subsection{Fuzzy concepts}

In (Thakur, Dewangan, and Thakur 2014) a novel hybrid transform and neuro fuzzy environment based gray image compression technique is proposed for providing an optimal image compression. The experimental results show that when compared to the traditional JPEG and JPEG2K techniques, the suggested compression technique minimized the Mean Square Error (MSE). In(Thakur and Thakur 2014) a fuzzy based soft hybrid JPEG standard is proposed for deploying an effective gray image compression codec. When compared to the traditional JPEG and JPEG2K standards the proposed fuzzy based soft hybrid JPEG technique provides higher compression ratio, increased image quality, higher compression ratio.

\subsection{Set Partitioning in Hierarchical Trees (SPIHT) algorithm}

In (A. Mallaiah 2012), the SPIHT algorithm with Huffman encoder is proposed for image compression. To improve the quality of the images, the Retinex algorithm is exploited. The suggested algorithm consumes less number of bits for the data transmission. The exploitation of Retinex algorithm at the receiver end enhanced the vision and quality of the images. The advantages of the SPIHT algorithm are faster results, simple and effective. The performance of various wavelets is compared using SPIHT algorithm in (Sadashivappa 2011). Prior to the application of the wavelet transform, the RGB component of the images are converted into $\mathrm{YCbCr}$. From the experimental results it is clear that the suggested algorithm provides an optimal PSNR and HVS property.

\subsection{Principal Component Analysis (PCA) based method}

In (Seema Kalangi 2013), a DWT based technique is proposed for the video compression. The 3D video is initially converted into 2D transformation for preventing the motion recompense step. By performing the video segmentation, visual change estimation and object tracking, the operations of the PCA based video representation algorithm is enabled. In (Khalilian and Bajic 2013) an empirical PCA-based decoding method is suggested for video watermarking. The suggested technique embeds the data in the LL subband of the wavelet coefficients. Based on the comparison among various elements of the initial principle component, the decoding is performed.

\section{EMBEDDING TECHNIQUES}

The embedding techniques for the video frames are classified into the following types,

- $\quad$ Digital watermarking

- Data hiding algorithms

\subsection{Digital watermarking}

Digital watermarking is defined as the process of introducing digital signal into the digital content. The steps involved in the digital watermarking process is represented in Fig.2.

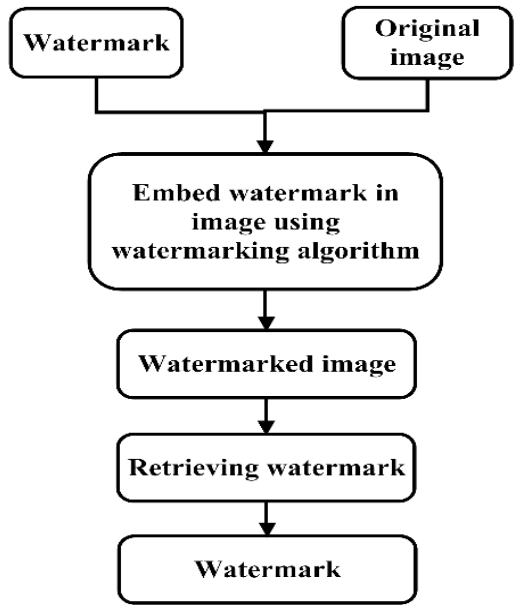

Fig 2: Overall process involved in the digital watermarking

Initially, the original image and the watermark image are embedded using the watermarking algorithm. After the embedding process, the watermarked image is obtained. On retrieving the watermarked image, the watermark and the original images are separated. An effective digital watermarking should have the following requirements,

- $\quad$ Robust

- Transparent

- Capability to withstand alterations and distortions.

- $\quad$ Efficient store and transmit

Fig. 3 represents the categorization of the digital watermarking techniques. 


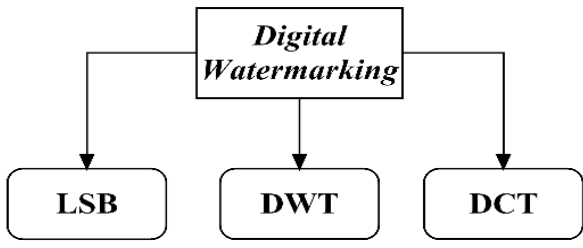

Fig 3: Types of digital watermarking

\subsubsection{Least Significant Bit (LSB)}

The LSB approach embeds the information in the cover image. The steps of the LSB technique are illustrated as follows,

\section{Steps involved in LSB}

Step 1: At first, the RGB image is converted to the gray scale image.

Step 2: Estimate the double precision of the image

Step 3: Shift the most significant bits to low significant bits of the watermark image

Step 4: Convert the least significant bits of the host image to zero.

Step 5: Add the shifted version of the watermarked image to modified host image.

In (Chopra et al. 2012) an LSB based digital image watermarking is proposed for the gray scale images. By exploiting the LSB algorithm, the message is embedded into the image. From the experimental results it is clear that the proposed watermarking algorithm provides optimal Mean Square Error (MSE) and Peak-Signal-to-Noise Ratio (PSNR) values. In (Singh, Shaw, and Alam 2015) the image security using LSB is analyzed. Further, the impact of noise in the images is analyzed. The analysis of noise impact and image security shows that the presence of noise attack in the watermarked images introduces an effect on the watermark image.

\section{Merits of LSB}

According to (Gurpreet Kaur 2013), the advantages of the LSB are as follows,

- $\quad$ Easy for deployment

- $\quad$ Simple

- Maximizes the visual fidelity of the images

\subsubsection{Discrete Wavelet Transform (DWT)}

The DWT is a time domain based analysis method with a fixed window size. The DWT for the image processing decomposes the image into four frequency bands such as LL, $\mathrm{LH}, \mathrm{HL}$ and HH. Among the bands, the LL band belongs to the low frequency district and the other three bands belong to the high frequency districts. The application of DWT transformation for the low frequency component information produces the sub-level frequency district information. According to (Senthil Nathan.M 2013) the two dimensional image after the application of three DWT decomposition is depicted in Fig.4. The figure represents the $\mathrm{L}$ as low pass filter and $\mathrm{H}$ as high pass filter. The HL1, LH1, HH1 are the frequency districts of the original image. The low-frequency district information are also decomposed into four districts such as LL2, HL2, LH2 and HH2. Generally, the signal information related to the original image are placed in the frequency districts such as $\mathrm{LH}, \mathrm{HL}$ and $\mathrm{HH}$ whereas the low frequency district information is closer to the original image.
Fig 4: Image decomposed using DWT

\begin{tabular}{|c|c|c|c|}
\hline LL3 & HL3 & HL2 & HL1 \\
\cline { 1 - 2 } LH3 & HH3 & \\
\cline { 1 - 2 } LH2 & HH 2 & \\
\cline { 1 - 2 } LH1 & HH1 \\
\hline \multicolumn{2}{|c|}{ H. } \\
\hline
\end{tabular}

According to (Shivani Khosla 2014) the precise formula for the first average sub signal is depicted as follows,

$$
a_{n}=\frac{f_{2 n-1}+f_{2}}{\sqrt{2}}, n=1,2,3, \ldots N / 2
$$

where, $\mathrm{N}$ represents the length of the signal. The overall steps involved in the DWT based watermarking are illustrated below,

Steps involved in DWT
Step 1: The original image is decomposed into multiple sub-
bands using DWT.
Step 2: The subband that is suitable for the embedding
watermark is selected.
Step 3: By exploiting the watermark image, the wavelet
coefficients are selected.
Step 4: Once the embedding process is complete, the
watermarked image is obtained.

In (Kashyap and Sinha 2012) a 3-level DWT is proposed for the robust image watermarking technique. By exploiting the alpha bending technique, the proposed technique embeds the low frequency sub-band of the cover image. The suggested technique provides optimal PSNR and MSE values. In (Deb et al. 2012) the DCT and DWT watermarking techniques are integrated with the low frequency watermarking. The suggested DCT based watermarking provides compression and the DWT based watermarking provides scalability. The suggested watermarking technique embeds the low frequency band of each DCT block of the selected DWT sub-band. When compared to the DCT and DWT based approaches, the proposed approach provides optimal image quality and robustness.

Advantages of DWT

- The original signal can be easily reconstructed using inverse wavelet transformation

- Provides position information by decomposing the original signal into wavelet transform.

\subsubsection{Discrete Cosine Transform (DCT)}

The DCT technique exploits the cosine waveform for converting the signal from the spatial domain to the frequency domain. The DCT represents the image as the sum of varying magnitudes and frequencies. According to (Navnidhi Chaturvedi 2012) the DCT coefficients are computed as follows,

$y(u, v)=$

$\sqrt{\frac{2}{M}} \sqrt{\frac{2}{N}} \alpha_{u} \alpha_{v} \sum_{u=0}^{M-1} \sum_{v=0}^{N-1} x(m, n) \cos \frac{(2 m+1) u \pi}{2 M} \cos \frac{(2 n+1) v \pi}{2 N}$ (2)

Where, alpha $\mathrm{u}$ and alpha $\mathrm{v}$ are computed as follows,

$$
\alpha_{u}=\left\{\begin{array}{l}
\frac{1}{\sqrt{2}} \mathrm{u}=0 ; u=1,2 \ldots N-1 \\
1
\end{array}\right.
$$




$$
\alpha_{v}=\left\{\begin{array}{l}
\frac{1}{\sqrt{2}} \mathrm{v}=0 ; \mathrm{v}=1,2 \ldots N-1 \\
1
\end{array}\right.
$$

On the receiver end, the inverse DCT operations is used for reconstructing the image.

$$
\begin{aligned}
& y(u, v) \\
& =\sqrt{\frac{2}{M}} \sqrt{\frac{2}{N}} \alpha_{u} \alpha_{v} \sum_{u=0}^{M-1} \sum_{v=0}^{N-1} x(m, n) \cos \frac{(2 m+1) u \pi}{2 M} \cos \frac{(2 n+1) v \pi}{2 N}
\end{aligned}
$$

In (Suhail and Obaidat 2003) a DCT based digital watermarking algorithm is suggested for securing the multimedia data. At first, the Voronoi diagram and feature extraction points are used for segmenting the image into multiple portions. The DCT domain is embedded with the image segments using real numbers represented as a pseudorandom sequence. Experimental results prove that the suggested algorithm achieves optimal compression ratio and robustness. In (Pan, Huang, and Chang 2013) a DCT based two dimensional linear discriminant analysis is proposed for watermarking the color images. Initially, the color image is transformed into YIQ color space then the transformed image is transformed into frequency domain by DCT algorithm. The suggested technique provides optimal robustness against the various attacks.

\section{Advantages of DCT}

- DCT has stronger energy compaction property

- All information are concentrated on the low frequency components of DCT.

- The high frequency components in the images are easily prevented.

\subsection{Data hiding techniques}

Data hiding is the process of encrypting the original image using a standard cipher with an encryption key.

\subsubsection{Data hiding of H.264/AVC video stream}

In (Ma et al. 2010) an efficient readable data-hiding algorithm is suggested for embedding the data into the quantized DCT coefficients. The distortion introduced by the embedding process are addressed using $4 \times 4$ DCT block. The distortion drift is prevented using the intra-frame prediction directions. The suggested algorithm increases the embedding capacity and decreases the visual distortion. In (Xu, Wang, and Shi 2014) a novel data hiding scheme is proposed for the H.264/AVC video stream. The suggested scheme has three components such as H.264/AVC video encryption, data embedding, and data extraction. Based on the properties of theH.264/AVC, the code words of the intra-prediction modes, code words of the motion vector differences and code words for the residual coefficients are encrypted with the stream ciphers. In (Li, Chen, and Zhao 2010) an H.264 encoded video sequence based method is proposed for hiding the data. By choosing the appropriate quantization and transformation coefficients, the desirable data is hidden. The hidden data are then recovered using the appropriate data recovering process. Further, they are extracted from the encoded stream.

\subsubsection{Data hiding techniques for MPEG video}

In (Wong et al. 2009) a novel data hiding method is proposed for maintaining the image quality of the host video. As the modified video reconstructs the original video in the video playback process, the suggested method is considered to be reversible. By exploiting the proposed method, the issues related to the video bit stream size increment are efficiently addressed. In (Shanableh 2012) two data hiding approaches are proposed for compressing the MPEG video. The first approach modulates the quantization scale of the constant bitrate video for hiding the messages and the second data hiding approach exploits the macro block ordering for hiding the message bits. By exploiting the content of the image, the macro blocks are allocated to the arbitrary slice groups. Thus, the proposed approaches produce optimal message payload with minimal distortion and compression overhead.

\section{PROPOSED WORK}

The overall flow of the suggested block code formation framework is represented in Fig.4. The suggested framework is used for embedding the consecutive video frames.

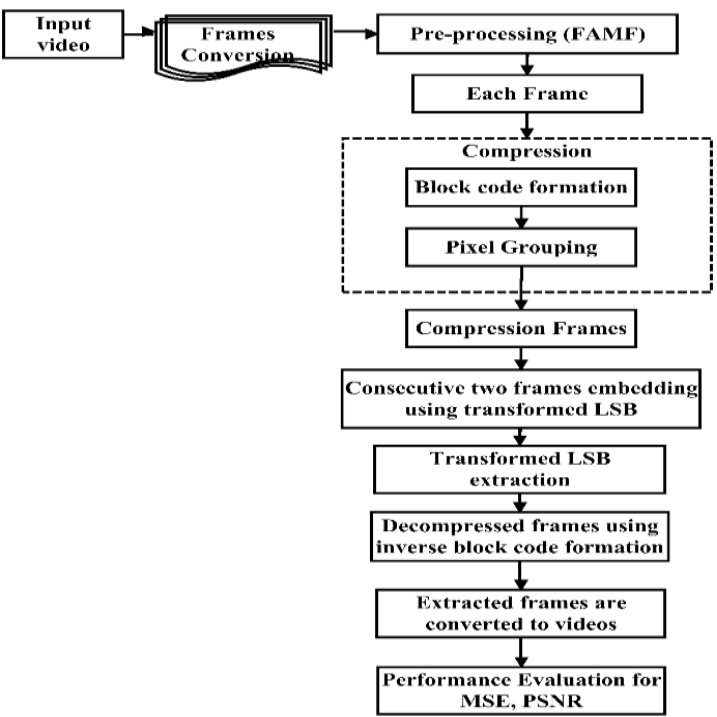

Fig 5: Overall flow of the suggested block code formation framework

At first, the input video will be split into multiple frames then each frame will be preprocessed using Fuzzy Adaptive Median Filter (FAMF). During the pre-processing process, the noises present in the frames will be eliminated. Before embedding the frames they are compressed using block wise pixel grouping. The suggested compression process splits the images into patches and estimates the recurrent pixels and location for all the patches. The estimated pixel values will be placed previous to the pixel value. The same process is repeated for the entire image. The output of each patch is considered as a code. Once all the frames are compressed, the Least Significant Bit (LSB) embedding technique embeds the compressed frames. On the receiver end, the compressed frames are decompressed using inverse block code formation. The decompressed frames are then converted back to the videos.

\section{CONCLUSION}

Video compression and embedding techniques are exploited for providing an efficient data transfer and maintenance of data security during transmission. In this paper, various image compression techniques such as fuzzy concepts, SPIHT algorithm, and PCA based methods are analyzed. Further, the digital embedding techniques such as digital watermarking and data hiding algorithms are surveyed. From the survey results, it is clear that the existing compression techniques do not efficiently restore the compressed images, the pixel information is lost during the transformations. Further, the traditional compression algorithms increase the time 
complexity and computational complexity and do not provide a satisfactory image/video security. Thus, to address all these issues a block code formation framework is proposed. The suggested framework exploits a block wise pixel grouping technique for performing the compression. During the compression process, the images are split into multiple patches. The recurrent location of the pixels is identified for each patch. The estimated pixel locations are placed prior to the pixel value for the entire image. After compressing the frames, the LSB algorithm performs the embedding process. The superiority of the proposed framework will be validated using the metrics such as PSNR, MSE and SSIM.

\section{REFERENCES}

[1] Mallaiah, S. K. Shabbir, T. Subhashini. 2012. "An Spiht Algorithm With Huffman Encoder For Image Compression And Quality Improvement Using Retinex Algorithm." International Journal of Scientific \& Technology Research no. 1 (5):45-49.

[2] Chopra, Deepshikha, Preeti Gupta, Gaur Sanjay, and Anil Gupta. 2012. "LSB based digital image watermarking for gray scale image." IOSR Journal of Computer Engineering (IOSRJCE) ISSN:2278-0661.

[3] Deb, Kaushik, Md Sajib Al-Seraj, Mohammed Moshiul Hoque, and Md Iqbal Hasan Sarkar. 2012. Combined DWT-DCT based digital image watermarking technique for copyright protection. Paper read at 7 th International Conference on Electrical \& Computer Engineering (ICECE).

[4] Gurpreet Kaur, Kamaljeet Kaur. 2013. "Image Watermarking Using LSB." International Journal of Advanced Research in Computer Science and Software Engineering no. 3 (4):858-861.

[5] Kashyap, Nikita, and GR Sinha. 2012. "Image watermarking using 3-level discrete wavelet transform (DWT)." International Journal of Modern Education and Computer Science no. 4 (3):50.

[6] Khalilian, Hanieh, and Ivan V Bajic. 2013. "Video watermarking with empirical PCA-based decoding." IEEE Transactions on Image Processing no. 22 (12):4825-4840.

[7] Khan, Asifullah, Ayesha Siddiqa, Summuyya Munib, and Sana Ambreen Malik. 2014. "A recent survey of reversible watermarking techniques." Information sciences no. 279:251-272.

[8] Li, Y., H. x. Chen, and Y. Zhao. 2010. A new method of data hiding based on H.264 encoded video sequences. Paper read at IEEE 10th International Conference on Signal Processing (ICSP), 24-28 Oct. 2010.

[9] Ma, Xiaojing, Zhitang Li, Hao Tu, and Bochao Zhang. 2010. "A data hiding algorithm for H. 264/AVC video streams without intra-frame distortion drift." IEEE Transactions on Circuits and Systems for Video Technology no. 20 (10):1320-1330.

[10] Navnidhi Chaturvedi, Dr.S.J.Basha. 2012. "Comparison of Digital Image watermarking Methods DWT \& DWTDCT on the Basis of PSNR." International Journal of Innovative Research in Science, Engineering and Technology no. 1 (2):147-153.

[11] Pan, I-Hui, Ping Sheng Huang, and Te-Jen Chang. 2013. "DCT-Based Watermarking for Color Images via TwoDimensional Linear Discriminant Analysis." In Information Technology Convergence, 57-65. Springer.

[12] Sadashivappa, K.V.S Anand Babu, Dr. Srinivas 2011. "Color Image Compression using SPIHT Algorithm." International Journal of Computer Applications no. 16 (7):34-42.
[13] Seema Kalangi, Veeraiah Maddu, Sreenivasa Ravi Kavuluri. 2013. "A Novel Approach of Low Complexity DWT/PCA Based Video Compression Method." International Journal of Engineering Science and Innovative Technology (IJESIT) no. 2 (3):159-168.

[14] Senthil Nathan.M , Pandiarajan.K, Baegan.U. 2013. "Digital Image Watermarking Basics " IOSR Journal of Electronics and Communication Engineering (IOSR$J E C E)$ no. 8 (1):07-11.

[15] Shanableh, T. 2012. "Data Hiding in MPEG Video Files Using Multivariate Regression and Flexible Macroblock Ordering." IEEE Transactions on Information Forensics $\begin{array}{llll}\text { and } & \text { Security no. } 7 \text { (2):455-464. doi: }\end{array}$ 10.1109/TIFS.2011.2177087.

[16] Shivani Khosla, Paramjeet Kaur 2014. "Secure Data Hiding Technique Using Video Steganography and Watermarking." International Journal of Computer Applications no. 95 (20):7-12.

[17] Singh, Ranjeet Kumar, Dilip Kumar Shaw, and M Javed Alam. 2015. "Experimental Studies of LSB Watermarking with Different Noise." Procedia Computer Science no. 54:612-620.

[18] Suganya.G, Mahesh.K. 2014. "A Survey: Various Techniques of Video Compression." International Journal of Engineering Trends and Technology (IJETT) no. 7 (1):10-12.

[19] Suhail, M. A., and M. S. Obaidat. 2003. "Digital watermarking-based DCT and JPEG model." IEEE Transactions on Instrumentation and Measurement no. 52 (5):1640-1647. doi: 10.1109/TIM.2003.817155.

[20] Thakur, S, Nilesh Kumar Dewangan, and Kavita Thakur. 2014. A Highly Efficient Gray Image Compression Codec Using Neuro Fuzzy Based Soft Hybrid JPEG Standard. Paper read at Proceedings of Second International Conference "Emerging Research in Computing, Information, Communication and Applications" ERCICA.

[21] Thakur, Vikrant Singh, and Kavita Thakur. 2014. Design and Implementation of a highly efficient gray image compression codec using fuzzy based soft hybrid JPEG standard. Paper read at International Conference on Electronic Systems, Signal Processing and Computing Technologies (ICESC).

[22] Wong, KokSheik, Kiyoshi Tanaka, Koichi Takagi, and Yasuyuki Nakajima. 2009. "Complete video qualitypreserving data hiding." IEEE Transactions on Circuits and Systems for Video Technology no. 19 (10):14991512.

[23] Xu, Dawen, Rangding Wang, and Yun Q Shi. 2014. "Data hiding in encrypted H. 264/AVC video streams by codeword substitution." IEEE Transactions on Information Forensics and Security no. 9 (4):596-606.

\section{AUTHOR PROFILE}

K.Rajalakshmi is pursing Ph.D degree Dept.of.Computer Science and EnggAlagappa University,Karaikudi

Dr.K.Mahesh ,Professor, Dept.of Computer Science\& Engg , Alagappa University,Karaikudi 